\title{
Rapid detection of Babesia motasi responsible for human babesiosis by cross-priming amplification combined with a vertical flow
}

\author{
Jinming Wang ${ }^{1}$, Shandian Gao', Shangdi Zhang², Xin He'1, Junlong Liu', Aihong Liu', Youquan Li', \\ Guangyuan Liu', Jianxun Luo ${ }^{1}$, Guiquan Guan ${ }^{1 *}$ and Hong Yin ${ }^{1,3^{*}}$
}

\begin{abstract}
Background: Babesia motasi is known as an etiological agent of human and ovine babesiosis. Diagnosis of babesiosis is traditionally performed by microscopy, examining Giemsa-stained thin peripheral blood smears. Rapid detection and accurate identification of species are desirable for clinical care and epidemiological studies.

Methods: An easy to operate molecular method, which requires less capital equipment and incorporates crosspriming amplification combined with a vertical flow (CPA-VF) visualization strip for rapid detection and identification of B. motasi.

Results: The CPA-VF targets the 185 rRNA gene and has a detection limit of $50 \mathrm{fg}$ per reaction; no cross reaction was observed with other piroplasms infective to sheep or Babesia infective to humans. CPA-VF and real-time (RT)-PCR had sensitivities of $95.2 \%$ (95\% confidence interval, Cl 78.1-99.4\%) and 90.5\% (95\% Cl 72-97.6\%) and specificities of 95.8 (95\% Cl 80.5-99.5\%) and 97.9 (95\% Cl 83.5-99.9\%), respectively, versus microscopy and nested (n) PCR combined with gene sequencing. The clinical performance of the CPA-VF assay was evaluated with field blood samples from sheep ( $n=340)$ in Jintai county, Gansu Province, and clinical specimens $(n=492)$ obtained from patients bitten by ticks.
\end{abstract}

Conclusions: Our results indicate that the CPA-VF is a rapid, accurate, nearly instrument-free molecular diagnostic approach for identification of B. motasi. Therefore, it could be an alternative technique for epidemiological investigations and diagnoses of ovine and/or human babesiosis caused by B. motasi, especially in resource-limited regions.

Keywords: Ovine babesiosis, Human babesiosis, Babesia motasi, Cross-priming amplification, Vertical flow visualization strip, Detection, Identification

\footnotetext{
*Correspondence: guanguiquan@caas.cn; yinhong@caas.cn

${ }^{1}$ State Key Laboratory of Veterinary Etiological Biology, Key Laboratory of Veterinary Parasitology of Gansu Province, Lanzhou Veterinary Research Institute, Chinese Academy of Agricultural Science, Xujiaping 1, Lanzhou, Gansu 730046, People's Republic of China

Full list of author information is available at the end of the article
}

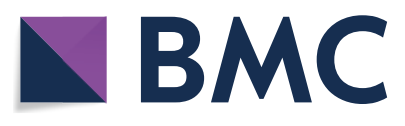

(c) The Author(s) 2020. This article is licensed under a Creative Commons Attribution 4.0 International License, which permits use, sharing, adaptation, distribution and reproduction in any medium or format, as long as you give appropriate credit to the original author(s) and the source, provide a link to the Creative Commons licence, and indicate if changes were made. The images or other third party material in this article are included in the article's Creative Commons licence, unless indicated otherwise in a credit line to the material. If material is not included in the article's Creative Commons licence and your intended use is not permitted by statutory regulation or exceeds the permitted use, you will need to obtain permission directly from the copyright holder. To view a copy of this licence, visit http://creativeco mmons.org/licenses/by/4.0/. The Creative Commons Public Domain Dedication waiver (http://creativecommons.org/publicdomain/ zero/1.0/) applies to the data made available in this article, unless otherwise stated in a credit line to the data. 


\section{Background}

Babesiosis, caused by protozoan pathogens of the genus Babesia infective to humans, domestic and wild animals, is one of the emerging and re-emerging tick-borne disease in the tropical and subtropical regions of the world [1]. It causes a wide spectrum of clinical signs which range from mild fever to serve anemia, haemoglobinuria and even death. Given increasing reports of human babesiosis, great attention has been paid to this emerging human disease $[2,3]$. Predominately, three Babesia spp., Babesia microti, B. divergens and B. duncani, have been described to be involved in human infections in the USA, Europe and Asia $[4,5]$. Recently, two newly emerging Babesia species, named as B. motasi and B. crassa, which were previously reported as causative agents of ovine babesiaosis, have been sporadically reported in cases of human babesiosis in Asia [6-10]. As a causative agent responsible for human babesiosis, the first case caused by B. motasi-like was reported in Korea in 2005 [6]. Recently, a 70-year-old man in Korea was diagnosed as infected with B. motasi [7].

In China, four strains of B. motasi (B. motasi Lintan, $B$. motasi Tianzhu, B. motasi Ningxian and B. motasi Hebei) are responsible for ovine babesiosis, and have been isolated from different endemic areas by the Vector and Vector-Borne Diseases (VVBD) Laboratory, Lanzhou Veterinary Research Institute (LVRI) [11-13]. Epidemiological studies have revealed that B. motasi infections have a wide distribution in sheep, goats, and vector ticks across China, according to molecular detection and serological analysis [14-16]. Given that it poses a severe threat to public health, rapid and accurate detection of $B$. motasi infection is important for performing epidemiological studies and providing appropriate clinical management. Several methods, based on molecular techniques that detect the presence of $B$. motasi genomic DNA, have been extensively accepted as the usual strategies for diagnosis of $B$. motasi infection. These methods, including polymerase chain reaction (PCR), RT-PCR, reverse line blot (RLB), and loop-mediated isothermal amplification (LAMP), require costly instruments and skilled personnel to perform the procedures, which has restricted their wide application in clinical care, infection control, and epidemiological studies [15, 17, 18].

Cross-priming amplification (CPA), a novel isothermal amplification technique, was developed as an alternative methodology for disease diagnosis in endemic areas where limited resources were available [19]. This approach has been applied to detection of a number of animal and plant pathogens, such as bacteria, viruses, and herbal products, with high specificity and sensitivity [20-23]. Given that it is an effective detection technique for reliable diagnosis of pathogen infection, in the present study a novel CPA targeting the $18 S$ rRNA gene was established for on-site detection of $B$. motasi infection. The labeled products from the CPA can be detected using a VF strip to visualize the specific amplicon of $B$. motasi.

\section{Methods}

\section{Primer design}

Babesia motasi specific primers for CPA were designed using the sequence alignments of the $18 S$ rRNA gene of Babesia spp. and Theileria spp. infective for sheep and humans (Table 1). A region that is conserved intra- $B$. motasi and variable among species was used as the target sequence for primer location. Two sets of primers and probes were designed using Primer Premier 5.0 software (Premier Biosoft International, Palo Alto, CA, USA); each set of primers and probes was composed of two displacement primers (BLT-5 s and BLT-4a), one cross primer (BLT-2 s1a), and two detector primers (BLT-2s and BLT-3 s). The detector primer (BLT-2 s) was labeled with biotin at the $5^{\prime}$-end and the BLT- $3 \mathrm{~s}$ was labeled with fluorescein isothiocyanate (FITC) at the $5^{\prime}$-end. The cross primer was composed of the BLT- $2 \mathrm{~s}$ at the $5^{\prime}$-end and $1 \mathrm{a}$ at the $3^{\prime}$-end. These primers were synthesized by TsingKe Biotech Co., Ltd (Beijing, China).

\section{Blood samples}

Standard positive samples were obtained from sheep experimentally infected with $B$. motasi. Briefly, 16 6-month-old sheep that were divided into 4 groups (groups 1-4) containing the same number of experimental animals were purchased from Jingtai county, Gansu Province, China, and confirmed to be free of piroplasm infection by microscopy, RT-PCR, nPCR and ELISA assay $[14,18,24,25]$. Groups 1-4 were inoculated intravenously $10 \mathrm{ml}$ of cryopreserved blood infected with $B$. motasi Lintan, B. motasi Tianzhu, $B$. motasi Ningxian and $B$. motasi Hebei, respectively. When parasitemia reached $8-10 \%$, blood samples were collected into EDTA-coated tubes. Three intact sheep were inoculated with $50 \mathrm{ml}$ blood infected with either B. motasi Lintan/B. motasi Tianzhu/B. motasi Ningxian/B. motasi Hebei via the jugular vein. Blood from the jugular vein was collected every 2 days after Babesia inoculation. Negative blood samples were collected into EDTA coated tubes from randomly selected sheep in Jintai county, Gansu province, where B. motasi is not endemic. All blood samples were transported to the VVBD laboratory, LVRI in iceboxes and stored at $-20^{\circ} \mathrm{C}$ before DNA extraction.

Genomic DNA was extracted from $200 \mu \mathrm{l}$ of the above-mentioned blood samples using a commercial DNA extractions kit according to the manufacturer's 
instruction (QIAamp DNA Blood Mini Kit; Qiagen, Hilden, Germany).

\section{Optimization of the CPA-VF assay for B. motasi detection} Initially, we designed two sets of primers to develop a highly sensitive and specific method. The CPA amplification was performed in a final volume of $20 \mu$ l. Following optimization of the reaction, the final composition was as follows: $1.25 \mu \mathrm{M}$ each of displacement primer (BLT-5 s and BLT-4a); $7.5 \mu \mathrm{M}$ each of detector primer (BLT-2 $\mathrm{s}$ and BLT-3 s); $12.5 \mu \mathrm{M}$ of cross primer (BLT-2s1a); $6 \mathrm{mM}$ $\mathrm{MgSO}_{4}, 20 \mathrm{mM}$ Tris-HCl (pH 8.8); $10 \mathrm{mM} \mathrm{KCl;} 1 \mathrm{M}$ betaine; $8 \mathrm{U}$ Bst DNA polymerase (New England BioLabs, Ipswich, UK); $8 \mathrm{mM}$ deoxynucleotides triphosphates (dNTPs); $0.1 \%$ Triton X-100; and $2 \mu$ genomic DNA. The $\mathrm{CPA}$ reaction tubes were incubated at $63{ }^{\circ} \mathrm{C}$ for $60 \mathrm{~min}$, followed by $80{ }^{\circ} \mathrm{C}$ for $2 \mathrm{~min}$ to terminate the reaction. Finally, VF strips, purchased from Hangzhou Ustar Company (Hangzhou, China), were used to detect CPA products: $5 \mu \mathrm{l}$ of CPA products and $90 \mu \mathrm{l}$ of PBS were added to the sample pad. A reaction was identified as positive when both the test line and the control line were developed, whereas it was considered as negative when only the control line was developed.

Furthermore, CPA reactions were performed at different temperatures, ranging from $55{ }^{\circ} \mathrm{C}$ to $65{ }^{\circ} \mathrm{C}$, and various time settings, ranging from 40 to $100 \mathrm{~min}$. Subsequently, the amplified products were detected using VF strips.

\section{Specificity and sensitivity of the CPA assays}

Genomic DNAs of Theileria luwenshuni, T. uilenbergi, T. ovis, Babesia sp. Xinjiang and Babesia sp. Dunhuang were provided by VVBD. The specificity of the assay was evaluated using genomic DNA from $B$. motasi Lintan, B. motasi Tianzhu, B. motasi Ningxian, B. motasi

Table 1 The sequences of B. motasi CPA-VF primers and probes

\begin{tabular}{|c|c|c|}
\hline & Primer name & Sequence $\left(5^{\prime}-3^{\prime}\right)$ \\
\hline \multirow[t]{5}{*}{ Set one } & BLT-5 S & GCTAATTGTAGGGCTAATACAAG \\
\hline & BLT-2 s & FITC-CGATGCCTTTTGGCGGCG \\
\hline & BLT-3 s & Biotin-GCTTTTAAACCAATTGTTGG \\
\hline & BLT-2s1a & $\begin{array}{l}\text { CGATGCCTTTTGGCGGCGCGATTCGCAAGTTTA } \\
\text { TTATG }\end{array}$ \\
\hline & BLT-4a & CTTGAATGGAACATCGCTAA \\
\hline \multirow[t]{5}{*}{ Set two } & BLT-5 s & GGADWWDGTCCGKTTTTG \\
\hline & BLT-2 s & FITC-CTTAGAGGGACTCCTGC \\
\hline & BLT-3 s & Biotin-GCTTGAAGCGTGGGGT \\
\hline & BLT-2s1a & CTTAGAGGGACTCCTGCCAGACCTGTTATTGCCTT \\
\hline & BLT-4a & CGCCTGCCGTTCGACGATT \\
\hline
\end{tabular}

Hebei, Babesia sp. Xinjiang, Babesia sp. Dunhuang, T. luwenshuni, T. uilenbergi, T. ovis, B. divergens, B. duncani, and plasmid DNAs bearing the $18 S$ rRNA gene of B. microti (GenBank: KF410825) and B. crassa (GenBank: AY260176). To evaluate the assay's sensitivity, serial dilutions of genomic DNA from purified B. motasi Lintan merozoites were used as the template for CPA amplification using the following concentrations: $4 \mathrm{ng} / \mu \mathrm{l} ; 800 \mathrm{pg} /$ $\mu \mathrm{l} ; 160 \mathrm{pg} / \mu \mathrm{l} ; 32 \mathrm{pg} / \mu \mathrm{l} ; 6.4 \mathrm{pg} / \mu \mathrm{l} ; 1.28 \mathrm{pg} / \mu \mathrm{l} ; 0.256 \mathrm{pg} /$ $\mu \mathrm{l} ; 50 \mathrm{fg} / \mu \mathrm{l}$; and $10 \mathrm{fg} / \mu \mathrm{l}$. Each concentration of genomic DNA was tested in three independent experiments to ensure reproducibility of the CPA assay.

To evaluate the performance of the CPA-VF assay, its sensitivity and specificity were determined using standard positive samples (experimentally infected animals) and field collected negative samples, versus microscopy, RT-PCR, and nPCR targeting the $18 S$ rRNA combined with gene sequencing $[18,24,26]$.

\section{Clinical performance of the CPA-VF assay for clinical specimens}

Field blood samples were randomly collected from 340 sheep in Gansu Province, transported to VVBD, LVRI in iceboxes and stored at $-20{ }^{\circ} \mathrm{C}$ before DNA extraction. A total of 492 patients who lived in the Gannan Tibetan Autonomous Prefecture (Gansu Province) who had visited the Second Hospital of Lanzhou University for a tick bite in the past few months, between May 2017 and July 2019, were recruited. Blood samples collected from patients were tested using the CPA-VF assay to determine the infection status of $B$. motasi.

The clinical performance of the CPA-VF approach was evaluated with field blood samples collected from sheep and clinical specimens from patients with a history of tick bite.

\section{Results \\ Optimization of the CPA primers, reaction temperature and time}

Sequence alignment of the $18 S$ rRNA genes of piroplasms infective to sheep and goats available in NCBI showed that two regions are conserved intra-species and variable among species. The sequences and locations of the primers are presented in Table 1. The primers and probes of set one showed high specificity. Therefore, set one was used for subsequent experiments. To determine a suitable amplification temperature, the CPA reactions were incubated at $55-65{ }^{\circ} \mathrm{C}$ for $60 \mathrm{~min}$. The results showed that the assay could be performed at a wide range of temperatures, from 58 to $63{ }^{\circ} \mathrm{C}$. Changes in amplification temperature had a slight impact on the brightness of the bands, 
indicating that incubation temperature is significant for the CPA reaction. The optimal brightness of a red-purple band was observed in the VF strip at a temperature $61{ }^{\circ} \mathrm{C}$ (Fig. 1a).

The CPA amplification was conducted at $61{ }^{\circ} \mathrm{C}$ for 40-100 min. The results revealed that positive signs could be developed as early as $40 \mathrm{~min}$ after amplification; however, the brightness of the positive band was not strong as after $60 \mathrm{~min}$ and $80 \mathrm{~min}$. To provide a high sensitivity and time efficiency of the CPA assay, an amplification time of 60 min was used in B. motasi detection (Fig. 1b).

\section{Cross reaction of the developed CPA approach}

The CPA technique was evaluated by testing piroplasms infective for sheep, goats and humans. As shown in Fig. 2, no cross reaction was observed with other Babesia spp. and Theileria spp. These results demonstrated that the CPA assay is specific for identification of $B$. motasi (Fig. 2).

\section{Limit of detection of the CPA-VF assay}

The limit of detection of the CPA-VF assay was evaluated using fivefold serially diluted DNA from purified merozoites of $B$. motasi in three independent reactions from $4 \mathrm{ng} / \mu \mathrm{l}$ to $10 \mathrm{fg} / \mu \mathrm{l}$. The assay could detect as few as $50 \mathrm{fg} / \mu \mathrm{l}$ DNA of B. motasi (Fig. 1c). As shown, two bands on the VF strips were developed using $4 \mathrm{ng}$ to $50 \mathrm{fg}$ of genomic DNA, while only one band was observed with $10 \mathrm{fg}$ and negative control reactions.

\section{Sensitivity and specificity of CPA-VF}

A total of 42 samples of standard positive genomic DNA from experimentally infected sheep and 48 negative field samples were studied using both CPA-VF and RT-PCR (Table 2). The performance of CPA-VF and
RT-PCR is presented in Table 3. The CPA-VF and RTPCR had sensitivities of $95.2 \%$ (95\% confidence interval, CI 78.1-99.4\%) and 90.5\% (95\% CI 72-97.6\%) and specificities of $95.8 \%$ (95\% CI 80.5-99.5\%) and $97.9 \%$ (95\% CI 83.5-99.9\%) (Table 3). There was no significance difference between the performance of the CPAVF and RT-PCR methods.

\section{Evaluation of the CPA using samples from the field and clinical samples}

To evaluate the feasibility of using CPA-VF as an alternative approach for $B$. motasi detection, 340 whole blood samples from sheep and patients were subjected to CPAVF. The results of the CPA-VF assay showed that $3.8 \%$ $(13 / 340)$ of the samples collected from sheep in Gansu province were positive and the remaining samples were negative for $B$. motasi infection.

A total of 492 blood samples collected from patients bitten by ticks, who visited the hospital, were investigated for the presence of $B$. motasi. From the results of CPA-VF, three samples were positive for $B$. motasi infection. Furthermore, to validate the presence of $B$. motasi in these samples, RT-PCR and nPCR were also employed and the results showed that all samples were negative for B. motasi infection.

\section{Discussion}

To provide an effective diagnostic tool, a CPA method targeting the $18 S \mathrm{rRNA}$ sequences of $B$. motasi was successfully developed for rapidly detecting and discriminating B. motasi infection. The CPA assay could detect four strains of $B$. motasi: B. motasi Lintan, B. motasi Tianzhu, B. motasi Hebei, and B. motasi Ningxian. In addition, no cross-reaction was observed with piroplasms infective for sheep (Babesia sp. Xinjiang, T. uilenbergi, T. luwenshuni,

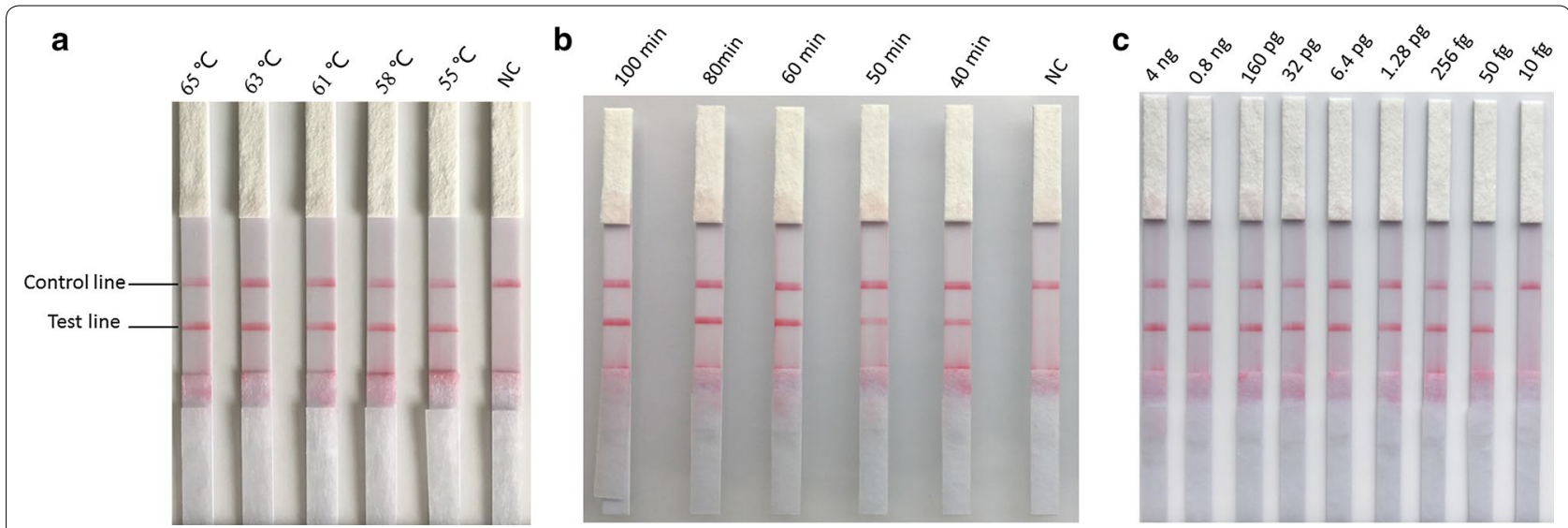

Fig. 1 Optimization of CPA reaction temperature and time and limit of detection evaluation of the CPA-VF assay with serial dilution of $B$. motasi DNA. a Reaction temperature of CPA-VF assay. $\mathbf{b}$ Reaction time of CPA-VF assay. $\mathbf{c}$ Detection limit of the CPA-VF 


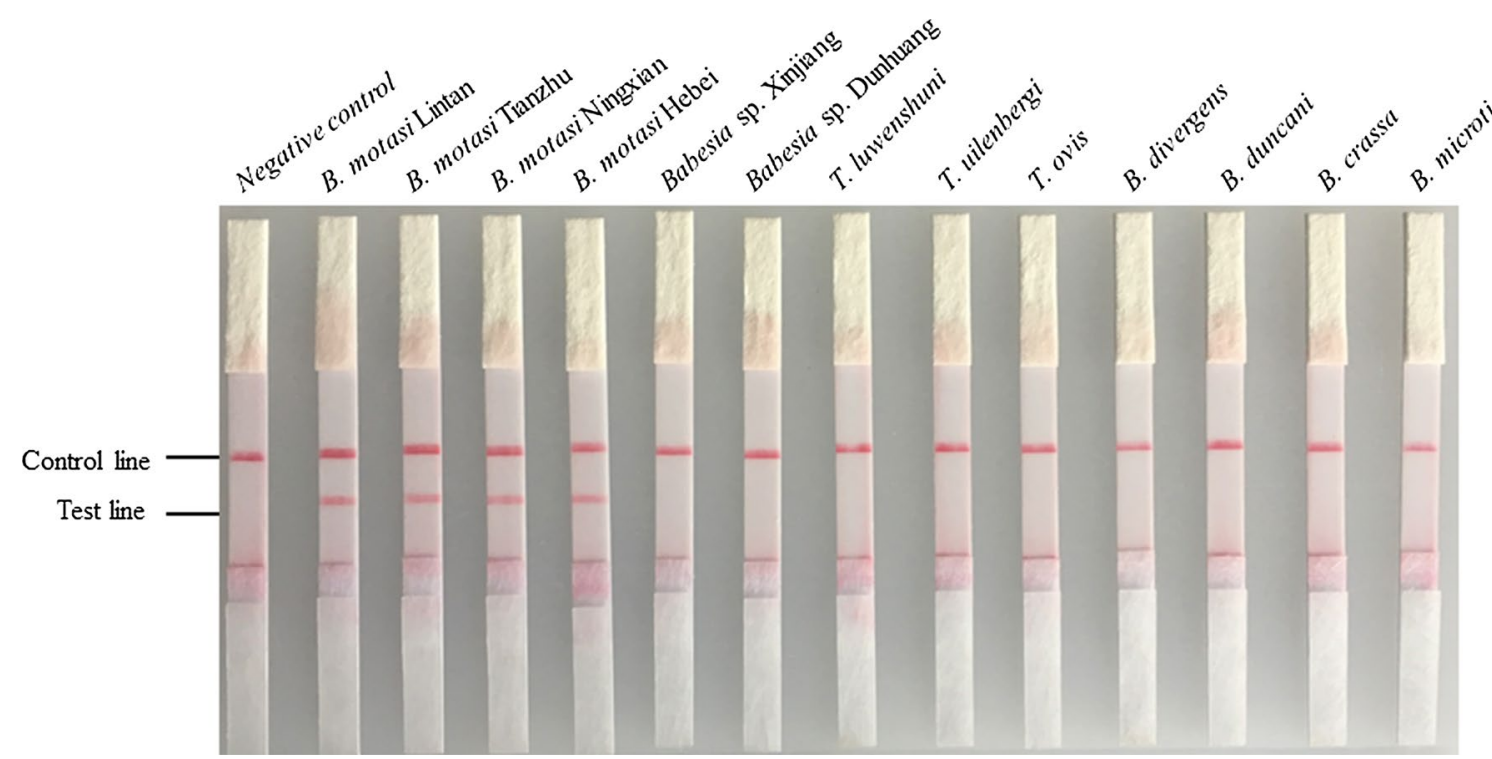

Fig. 2 Evaluation of specificity of the CPA-VF with genomic DNA (B. motasi Lintan, B. motasi Tianzhu, B. motasi Hebei, B. motasi Ningxian, Babesia sp. Xinjiang, Babesia sp. Dunhuang, T. uilenbergi, T. luwenshuni, T. ovis, A. ovis, B. duncani and B. divergens) and plasmids (B. microti and B. crassa)

Table 2 Standard positive and negative samples, confirmed by thin blood smear microscopy and nested PCR combined with gene sequencing

\begin{tabular}{lllllll}
\hline Result & B. motasi Lintan $(n)$ & B. motasiTianzhu $(n)$ & B. motasi Hebei $(n)$ & B. motasi Ningxian $(n)$ & Negative $(n)$ & Total $(n)$ \\
\hline Positive & 10 & 10 & 12 & 10 & 42 & 48 \\
Negative & 10 & 10 & 12 & 10 & 48 & 48 \\
Total & 10 & & & & 90
\end{tabular}

$n$ number of samples

T. ovis and $A$. ovis) and humans (B. duncani, B. divergens, $B$. microti and $B$. crassa). Further studies should be performed to investigate any potential cross-reactivity with other pathogens infective for humans using the CPA-VF approach developed herein.

In our present study, CPA-VF could detect as few as $50 \mathrm{fg}$ of genomic DNA from $B$. motasi per reaction, which was equal to approximately $50 \mu \mathrm{l}$ of $0.000,005 \%$ parasitized erythrocytes. The CPA reaction does not require expensive equipment and can be performed in a constant temperature block to maintain a reaction temperature of $61{ }^{\circ} \mathrm{C}$ for $60 \mathrm{~min}$. Furthermore, products generated by the CPA amplification can be detected using a VF strip, which only takes 2-5 min and is visible to the naked eye. Thus, the CPA assay is suitable for rapid, simple, and sensitive detection of $B$. motasi infection in limited-resource settings in endemic regions.
Table 3 The performance of the CPA-VF assay compared with that of RT-PCR

\begin{tabular}{lll}
\hline Result & \multicolumn{2}{l}{ Detection method } \\
\cline { 2 - 3 } & RT-PCR & CPA-VF \\
\hline True positive & 38 & 40 \\
False positive & 1 & 2 \\
True negative & 47 & 46 \\
False negative & 4 & 2 \\
Sensitivity (\%) & $90.5(72-97.6)$ & $95.2(78.1-99.4)$ \\
Specificity (\%) & $97.9(83.5-99.9)$ & $95.8(80.5-99.5)$ \\
\hline
\end{tabular}

To assess its suitability for clinical use, we conducted the first diagnostic study in clinical specimens and host animals using CPA-VF, comparing it with microscopy, RT-PCR, and nPCR combined with PCR product 
sequencing. The results from studies of a positive and negative panel revealed that CPA-VF has better sensitivity than that of RT-PCR. Because of its sensitivity, CPA-VF could be useful for the preliminary screening of low-level parasitemia. Our results demonstrate that excellent sensitivity was observed for the CPA-VF approach in comparison with that of RT-PCR. However, false positives that needed to be confirmed by microscopy should be noted with the CPA-VF assay. Three samples determined to be negative for piroplasm infection by $\mathrm{nPCR}$ were shown to present $B$. motasi infections by CPA-VF analysis. According to the clinical records, these three people presented virus-like or flu-like symptoms, clinical laboratory data showed they might be infected with bacteria (Additional file 1: Table S1). They were treated with azithromycin and cephalosporin and did not come back to hospital again. As these cases were outpatients we were not able to obtain enough information about the outcome of these patients. One limitation of this study was the small number of positive specimens, which were used to evaluate clinical performance. Further studies are needed regarding the implementation of this approach into clinical practice.

\section{Conclusions}

We successfully developed CPA-VF analysis for rapid and specific detection of $B$. motasi, with high sensitivity (95.2\%) and specificity (95.8\%). The developed CPA-VF assay does not require sophisticated equipment and has an easy nucleic acid detection system. The study provided a practical, easy-to-operate and alternative method for performing epidemiological and point-of-care diagnosis for B. motasi infection, although there should be some caution regarding false positives when CPA is used for clinical screening.

\section{Supplementary information}

Supplementary information accompanies this paper at https://doi. org/10.1186/s13071-020-04246-4.

Additional file 1: Table S1. Clinical information for three patients.

\section{Abbreviations}

CPA-VF: Cross-priming amplification combined with a vertical flow; LAMP: Loop-mediated isothermal amplification; RT-PCR: Real-time PCR; VVBD: Vectors and vector-borne diseases laboratory; LVRI: Lanzhou Veterinary Research Institute; nPCR: Nested PCR; RLB: Reverse line blot.

\section{Acknowledgements}

Not applicable.

\section{Authors' contributions}

HY, JLu and GG designed the study. JW performed the experiments, analyzed the results and wrote the manuscript. SG, SZ, JLi, XH, YL, GL and AL contributed reagents/materials/equipment. All authors read and approved the final manuscript.

\section{Funding}

This study was financially supported by the National Key Research and Development Program of China (2017YFD0501200; 2018YFD050230; 2017YFD0500904; 2017YFD0502300), 973 Program (2015CB150300), ASTIP (CAAS-ASTIP-2016-LVRI), NBCIS (CARS-37) and Jiangsu Co-innovation Center programme for Prevention and Control of Important Animal Infectious Disease and Zoonoses. Cuiying Scientific and Technological Innovation Program of Lanzhou University Second Hospital.

\section{Availability of data and materials}

Not applicable.

\section{Ethics approval and consent to participate}

The collection and manipulation of sheep blood samples was approved by the Animal Ethics Committee of the Lanzhou Veterinary Research Institute, Chinese Academy of Agricultural Sciences. All sampling procedures were handled in accordance with the Animal Ethics Procedures and Guidelines of the People's Republic of China (Permit No. LVRIAEC-2018-001). The study of clinical specimens was approved by the Ethics Committee of The Second Hospital of Lanzhou University (reference 2018A-046). All the procedures conducted were according to the Ethical Procedures and Guidelines of the People's Republic of China.

\section{Consent for publication}

Not applicable.

\section{Competing interests}

The authors declare that they have no competing interests.

\section{Author details \\ ${ }^{1}$ State Key Laboratory of Veterinary Etiological Biology, Key Laboratory of Veterinary Parasitology of Gansu Province, Lanzhou Veterinary Research Institute, Chinese Academy of Agricultural Science, Xujiaping 1, Lanzhou, Gansu 730046, People's Republic of China. ${ }^{2}$ Department of Clinical Labora- tory, The Second Hospital of Lanzhou University, Lanzhou, Gansu 730000, People's Republic of China. ${ }^{2}$ Jiangsu Co-Innovation Center for the Prevention and Control of Important Animal Infectious Disease and Zoonoses, Yangzhou University, Yangzhou 225009, People's Republic of China.}

Received: 20 January 2020 Accepted: 20 July 2020

Published online: 29 July 2020

\section{References}

1. Uilenberg G. International collaborative research: significance of tickborne hemoparasitic diseases to world animal health. Vet Parasitol. 1995:57:19-41.

2. Friedhoff KT. Tick-borne diseases of sheep and goats caused by Babesia Theileria or Anaplasma spp. Parassitologia. 1997:39:99-109.

3. Yin H, Luo J. Ticks of small ruminants in China. Parasitol Res. 2007;101(Suppl. 2):S187-9.

4. Vannier E, Krause PJ. Human babesiosis. N Engl J Med. 2012;366:2397-407.

5. Mueller I, Shakri AR, Chitnis CE. Development of vaccines for Plasmodium vivax malaria. Vaccine. 2015;33:7489-95.

6. Kim JY, Cho SH, Joo HN, Tsuji M, Cho SR, Park IJ, et al. First case of human babesiosis in Korea: detection and characterization of a novel type of Babesia sp. (KO1) similar to ovine babesia. J Clin Microbiol. 2007:45:2084-7.

7. Hong SH, Kim SY, Song BG, Rho JR, Cho CR, Kim CN, et al. Detection and characterization of an emerging type of Babesia sp. similar to Babesia motasi for the first case of human babesiosis and ticks in Korea. Emerg Microbes Infect. 2019;8:869-78.

8. Man SQ, Qiao K, Cui J, Feng M, Fu YF, Cheng XJ. A case of human infection with a novel Babesia species in China. Infect Dis Poverty. 2016;5:28.

9. Jiang JF, Zheng YC, Jiang RR, Li H, Huo QB, Jiang BG, et al. Epidemiological clinical, and laboratory characteristics of 48 cases of "Babesia venatorum" infection in China: a descriptive study. Lancet Infect Dis. 2015;15:196-203. 
10. Wang J, Zhang S, Yang J, Liu J, Zhang D, Li Y, et al. Babesia divergens in human in Gansu Province China. Emerg Microbes Infect. 2019;8:959-61.

11. Bai Q, Liu G, Liu D, Ren J, Li X. Isolation and preliminary characterization of a large Babesia sp. from sheep and goats in the eastern part of Gansu Province, China. Parasitol Res. 2002;88(Suppl. 1):S16-21.

12. Guan GQ, Yin H, Luo JX, Lu WS, Zhang QC, Gao YL, et al. Transmission of Babesia sp. to sheep with field-collected Haemaphysalis qinghaiensis. Parasitol Res. 2002;88(Suppl. 1):S22-4.

13. Liu AH, Yin H, Guan GQ, Schnittger L, Liu ZJ, Ma ML, et al. At least two genetically distinct large Babesia species infective to sheep and goats in China. Vet Parasitol. 2007;147:246-51.

14. Wang JM, Ma ML, Liu AH, Ren QY, Li AY, Liu ZJ, et al. A sero-epidemiological survey of Chinese Babesia motasi for small ruminants in China. Parasitol Res. 2013;112:2387-91.

15. Niu QL, Luo JX, Guan GQ, Ma ML, Liu ZJ, Liu AH, et al. Detection and differentiation of ovine Theileria and Babesia by reverse line blotting in China. Parasitol Res. 2009;104:1417-23.

16. Niu QL, Luo JX, Guan GQ, Liu ZJ, Ma ML, Liu AH, et al. Differentiation of two ovine Babesia based on the ribosomal DNA internal transcribed spacer (ITS) sequences. Exp Parasitol. 2009;121:64-8.

17. Guan GQ, Chauvin A, Luo JX, Inoue N, Moreau E, Liu ZJ, et al. The development and evaluation of a loop-mediated isothermal amplification (LAMP) method for detection of Babesia spp. infective to sheep and goats in China. Exp Parasitol. 2008;120:39-44.

18. Yang Q, Liu A, Liu J, Jifei Y, Youquan L, Zhijie L, et al. Molecular epidemiological investigation of ovine Babesia spp. in 10 provinces of China. Chinese Vet Sci. 2016;46:597-601.

19. $\mathrm{Xu} \mathrm{G}, \mathrm{Hu}$ L, Zhong $H$, Wang $H$, Yusa S, Weiss TC, et al. Cross priming amplification: mechanism and optimization for isothermal DNA amplification. Sci Rep. 2012;2:246.
20. Gao Y, Meng XY, Zhang HW, Luo YZ, Sun Y, Li YF, et al. Cross-priming amplification combined with immunochromatographic strip for rapid on-site detection of African swine fever virus. Sensor Actuat B-Chem. 2018;274:304-9.

21. Huo YY, Li GF, Qiu YH, Li WM, Zhang YJ. Rapid detection of prunus necrotic ringspot virus by reverse transcription-cross-priming amplification coupled with nucleic acid test strip cassette. Sci Rep. 2017;7:16175.

22. Wang Y, Wang Y, Ma A, Li D, Ye C. Rapid and sensitive detection of Listeria monocytogenes by cross-priming amplification of Imo0733 gene. FEMS Microbiol Lett. 2014;361:43-51.

23. Su ZD, Shi CY, Huang J, Shen GM, Li J, Wang SQ, et al. Establishment and application of cross-priming isothermal amplification coupled with latera flow dipstick (CPA-LFD) for rapid and specific detection of red-spotted grouper nervous necrosis virus. Virol J. 2015;12:149.

24. Yang JF, Li YQ, Liu ZJ, Liu JL, Guan GQ, Chen Z, et al. Molecular evidence for piroplasms in wild Reeves' muntjac (Muntiacus reevesi) in China. Parasitol Int. 2014;63:713-6.

25. Guan G, Ma M, Liu A, Ren Q, Wang J, Yang J, et al. A recently identified ovine Babesia in China: serology and sero-epidemiology. Parasitol Int. 2012;61:532-7.

26. Olmeda AS, Armstrong PM, Rosenthal BM, Valladares B, delCastillo A, deArmas F, et al. A subtropical case of human babesiosis. Acta Trop. 1997;67:229-34.

\section{Publisher's Note}

Springer Nature remains neutral with regard to jurisdictional claims in published maps and institutional affiliations.
Ready to submit your research? Choose BMC and benefit from:

- fast, convenient online submission

- thorough peer review by experienced researchers in your field

- rapid publication on acceptance

- support for research data, including large and complex data types

- gold Open Access which fosters wider collaboration and increased citations

- maximum visibility for your research: over 100M website views per year

At BMC, research is always in progress.

Learn more biomedcentral.com/submissions 